\title{
Molecular characterisation of patients with subtelomeric $22 q$ abnormalities using chromosome specific array-based comparative genomic hybridisation
}

\author{
David A Koolen ${ }^{1}$, William Reardon ${ }^{2}$, Elisabeth M Rosser ${ }^{3}$, Didier Lacombe ${ }^{4}$, Jane A Hurst ${ }^{5}$, \\ Caroline J Law ${ }^{6}$, Ernie MHF Bongers ${ }^{1}$, Conny M van Ravenswaaij-Arts ${ }^{1}$, Martijn AR Leisink ${ }^{7}$, \\ Ad Geurts van Kessel ${ }^{1}$, Joris A Veltman ${ }^{1}$ and Bert BA de Vries*, ${ }^{*}$

\begin{abstract}
${ }^{1}$ Department of Human Genetics, Radboud University Nijmegen Medical Centre, Nijmegen, The Netherlands;
${ }^{2}$ National Centre for Medical Genetics, Our Lady's Hospital for Sick Children, Crumlin, Dublin, Ireland; ${ }^{3}$ Department of Clinical Genetics, Institute of Child Health, London, UK; ${ }^{4}$ Department of Medical Genetics, CHU Pellegrin, Bordeaux, France; ${ }^{5}$ Department of Clinical Genetics, The Churchill Hospital, Oxford, UK; ${ }^{6}$ Wessex Regional Genetics Service, The Princess Anne Hospital, Southampton, UK; ${ }^{7}$ Department of Medical Physics and Biophysics, Radboud University Nijmegen Medical Centre, Nijmegen, The Netherlands
\end{abstract}

The 22q13 deletion syndrome is associated with global developmental delay, absent or delayed speech, and generalised hypotonia. In this study, the size and nature of $22 q 13$ deletions $(n=9)$ were studied in detail by high-resolution chromosome specific array-based comparative genomic hybridisation (array $\mathrm{CGH})$. The deletion sizes varied considerably between the different patients, that is, the largest deletion spanning $8.4 \mathrm{Mb}$ with the breakpoint mapping to $22 \mathrm{q} 13.2$ and the smallest deletion spanning $3.3 \mathrm{Mb}$ with the breakpoint mapping to $22 q 13.31$. In one case, a unique subtelomeric $3.9 \mathrm{Mb}$ deletion associated with a $2.0 \mathrm{Mb}$ duplication of $22 \mathrm{q} 13$ was observed, adding to a growing number of similar cases identified for other chromosome ends. Remarkably, this patient had signs suggestive of retinitis pigmentosa, which has never been reported before in the $22 q 13$ deletion syndrome. The identification of two pairs of recurrent proximal breakpoints on $22 q 13$ suggests that these specific regions may be prone to recombination, due to yet unknown genome architectural features. In addition to the copy number changes on $22 q 13$, a duplication of $\sim 330 \mathrm{~kb}$ on $22 q 11.1$ was observed and shown to be a genetic large-scale copy number variation without clinical consequences. The current study failed to reveal relationships between the clinical features and the deletion sizes. Global developmental delay and absent or severely delayed speech were observed in all patients, whereas hypotonia was present in $89 \%$ of the cases $(8 / 9)$. This study underscores the utility of array CGH for characterising the size and nature of subtelomeric deletions, such as monosomy $22 q 13$, and underlines the considerable variability in deletion size in the $22 q 13$ deletion syndrome regardless of the clinical phenotype.

European Journal of Human Genetics (2005) 13, 1019-1024. doi:10.1038/sj.ejhg.5201456;

published online 29 June 2005

Keywords: 22q13 deletion syndrome; array $\mathrm{CGH}$; mental retardation; submicroscopic; subtelomeric

*Correspondence: Dr BBA de Vries, Department of Human Genetics, 417, Radboud University Nijmegen Medical Centre, PO Box 9101, Nijmegen 6500 HB, The Netherlands. Tel: + 3124361 3946; Fax: + 3124366 8753;
E-mail: b.devries@antrg.umcn.nl

Received 25 February 2005; revised 9 May 2005; accepted 19 May 2005; published online 29 June 2005 


\section{Introduction}

Subtelomeric deletions are a common cause of mental retardation. ${ }^{1,2}$ In recent years, screening of subtelomeres for copy number changes in mentally retarded patients has resulted in the recognition of new distinct clinical entities, based on monosomy 1q, 2q, 9q, 14q, and 22q (reviewed in Vries et $\mathrm{ll}^{1}$ ) The latter entity is also referred to as the $22 \mathrm{q} 13$ deletion syndrome, of which to date at least 100 cases have been reported. ${ }^{3-6}$ Common features associated with this syndrome are mild-to-severe global developmental delay, absent or delayed speech, generalised hypotonia, and minor anomalies including dolichocephaly, ptosis, abnormal ears, relatively large hands, and dysplastic toenails. ${ }^{5}$

Here we describe a series of nine patients with monosomy 22q13 in which the size and the nature of the chromosome 22 deletions were studied in detail by highresolution chromosome-specific array-based comparative genomic hybridisation (array CGH). ${ }^{7}$

\section{Materials and methods Patients and DNA samples}

In total, nine patients with subtelomeric deletions of $22 q 13$ were included in this study. Three cases were previously published (case $2,{ }^{8}$ case $7^{9}$ and case $9^{10}$ ). DNA samples of the patients were derived from different clinical centres in France, Ireland, the United Kingdom, and the Netherlands (Nijmegen). Clinical information on the patients was obtained from the referring physician. DNA samples of all patients were analysed using array CGH. In one patient (case 3), the 22qter deletion was initially identified by routine chromosome analysis at a 550-band level and confirmed by fluorescence in situ hybridisation (FISH) [46, XY, del(22)(q13.2).ish del(22)(TUPLE + , ARSA-)]. Another deletion (case 4) resulted from a de novo translocation [46, XY, der(22)t(14;22)(q32.33;q13.31)pat. ish $\operatorname{der}(22)(\mathrm{N} 85 \mathrm{~A} 3-)]$, whereas the remaining cases were initially identified by FISH, using the following probes: N85A3 (cases 1-2, 5), ARSA (cases 6-8), D22S163 (case 8), or STS WI-941 and D22S39 (case 9).

\section{Array-based comparative genomic hybridisation} High-resolution chromosome 22 array CGH was performed. The microarray consisted of 350 positionally selected chromosome 22 BAC clones (BACPAC Resources), together with 1532 control clones located on other chromosomes. The chromosome 22-specific clones covered the long arm of chromosome $22(35.1 \mathrm{Mb})$ with an average spacing of one clone per $100 \mathrm{~kb}$. However, the actual resolution may be less in repeat dense regions. The average clone-insert size was $168 \mathrm{~kb}$, resulting in a 1.7-fold coverage of the long arm of chromosome 22. DOP-PCR products of the BAC clones were spotted in six-fold onto CMT-GAPScoated glass slides (Ultragaps, Corning) using an OmniGrid 100 arrayer (Genomic Solutions). All steps in the labelling, hybridisation, and data-analysis procedure were performed as described previously. ${ }^{11}$ In brief, equal amounts of patient and reference genomic DNAs were labelled by random priming with Cy3-dUTP or Cy5-dUTP (Amersham Biosciences). Labelled test- and reference DNAs were mixed with Cot-1 DNA (Roche), coprecipitated and resuspended in a hybridisation solution. After denaturation of probe and target DNA, hybridisation and posthybridisation washing procedures were performed using a GeneTac Hybridisation Station (Genomic Solutions), according to the instructions of the manufacturer. Fluorescence intensity images were acquired using an Affymetrix 428 scanner (Affymetrix), and analysed by Genepix Pro 5.1 (Axon Instruments).

\section{Statistical data analysis}

Data normalisation was performed by applying Lowess curve fitting with a smoothing factor of 0.3 as described by Vissers et $a l^{11}$ Copy number alterations were identified by using a Hidden Markov Model algorithm. Three hidden states represent normal, loss, and gain conditions. The probability to observe a $\log$ ratio given its hidden state is modelled by a Gaussian with SD 0.25 and mean $0,-0.4$ and 0.4 , respectively. The hidden state of adjacent clones is correlated with respect to their distance. The thresholded marginal probability of a clone's hidden state determines if it is normal, a loss or gain. In order to discriminate between causative aberrations and large-scale copy number variations (LCV) we used a data set of 72 normal individuals. The control population was tested by a genome-wide 32.477 clone BAC array which included the same clones as used for the chromosome 22 array.

\section{Results}

Seven patients with a submicroscopic 22qter deletion, one patient with an unbalanced translocation and one patient with a microscopically visible 22qter deletion were studied. In Table 1, the main characteristics of the patients are summarised. In addition, minor facial dysmorphisms were noted (Figure 1). High-resolution chromosome 22-specific array CGH confirmed the known copy number changes in all cases and delineated the specific aberrations in detail. Figure 2 shows two examples of chromosome 22 array CGH profiles. All deletions analysed included the most telomeric clones. Interstitial deletions in the subtelomeric region of the long arm of chromosome 22 were not observed. The deletion sizes identified in this study varied considerably between the different patients, that is, the largest deletion spanning $8.4 \mathrm{Mb}$ with breakpoint mapping to 22q13.2qter (68 clones, case 1) and the smallest deletion spanning $3.3 \mathrm{Mb}$ with breakpoints mapping to 22q13.31qter (27 clones, case 9) (Figure 3). Identical proximal breakpoints were localised in cases 2 and 3 (between RP11-786006 and RP11-236I15) and cases 5 and 6 (between RP11-766K21 and 
Table 1 Main characteristics of $22 q 13$ deletion cases presented in this study

\begin{tabular}{|c|c|c|c|c|c|c|c|c|c|}
\hline Patients & 1 & $2^{8}$ & 3 & $4^{\mathrm{a}}$ & 5 & 6 & $7^{9}$ & 8 & $9^{10}$ \\
\hline Deletion size $(\mathrm{Mb})$ & 8.4 & 7.7 & 7.7 & 6.6 & 5.9 & 5.9 & 5.0 & 3.9 & 3.3 \\
\hline Duplication size (Mb) & - & - & - & - & - & - & - & 2.0 & - \\
\hline \multicolumn{10}{|l|}{ Clinical characteristics } \\
\hline Sex & $\mathrm{F}$ & M & M & M & $\mathrm{F}$ & $\mathrm{F}$ & M & $\mathrm{F}$ & $\mathrm{F}$ \\
\hline Global developmental delay & + & + & + & + & + & + & + & + & + \\
\hline Normal to accelerated growth & + & + & + & + & + & + & + & - & + \\
\hline Absent/severely delayed speech & + & + & + & + & + & + & + & + & + \\
\hline Hypotonia & + & + & + & + & + & + & + & - & + \\
\hline Chewing behaviour & + & - & - & - & - & - & + & - & - \\
\hline Dolichocephaly & - & - & - & - & - & - & - & - & - \\
\hline Ptosis & - & - & - & - & - & - & - & - & - \\
\hline Prominent/dysplastic ears & + & + & + & + & + & + & + & + & - \\
\hline Prominent/pointed chin & + & - & - & + & + & + & + & - & - \\
\hline Relatively large, fleshy hands & + & - & - & - & + & + & + & + & - \\
\hline Abnormal toenail growth & + & + & + & - & + & - & + & - & - \\
\hline
\end{tabular}

$\mathrm{F}$, female; $\mathrm{M}$, male; +, feature present; - , feature absent.

${ }^{\mathrm{a}} 46, \mathrm{XY}, \operatorname{der}(22) \mathrm{t}(14 ; 22)$.
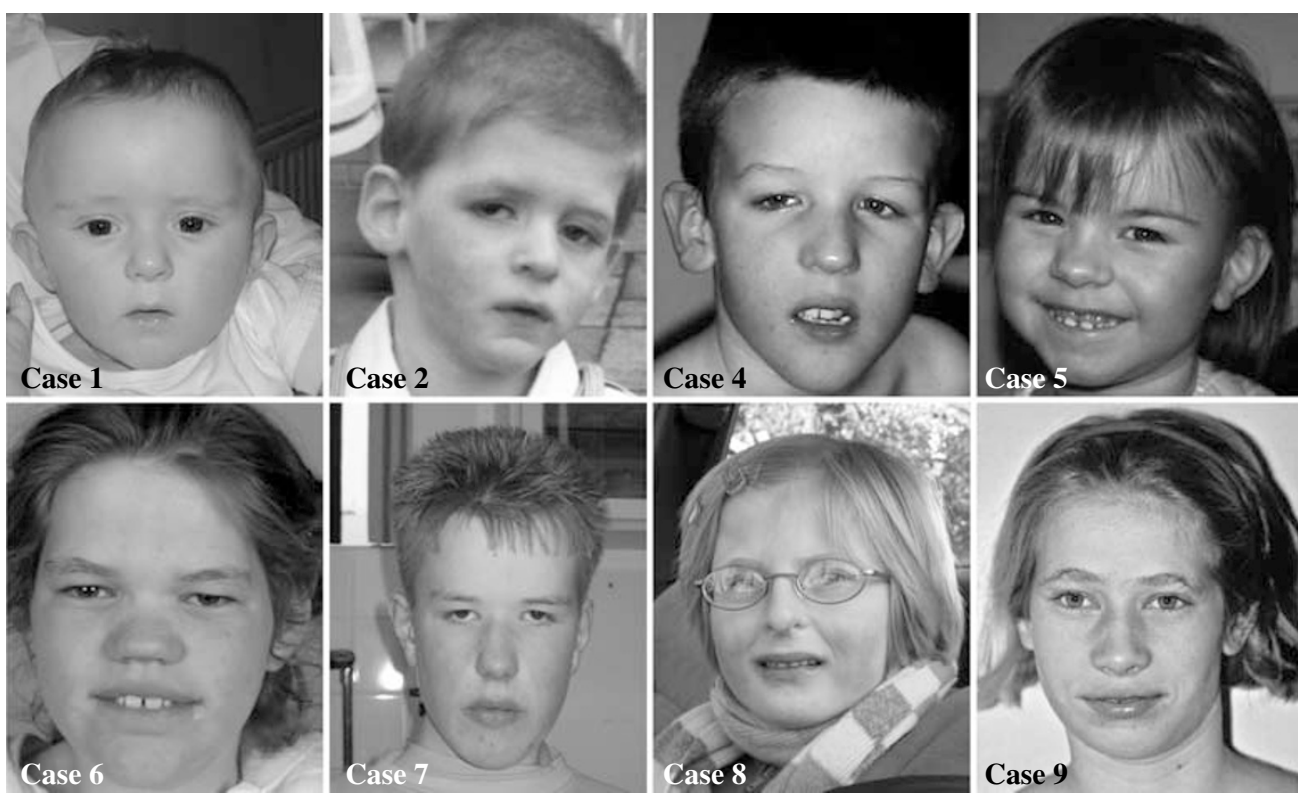

Figure 1 Patients with $22 q 13$ deletion syndrome. Note the known facial features of the $22 q 13$ deletion syndrome, prominent dysplastic ears (visible in cases 1, 2, 4 and 5), and a prominent/ pointed chin (cases 1 and 4-7).

RP11-49A20), resulting in deletion sizes of 7.7 and $5.9 \mathrm{Mb}$, respectively. In case 3 , a $\operatorname{del}(22)$ (q13.2qter) was originally identified by routine chromosome analysis. Array CGH analysis confirmed the karyotypic analysis and established the deletion size to $7.7 \mathrm{Mb}$. Case 4 carried a translocation between chromosomes 14 and 22 [46, XY, der(22)$\mathrm{t}(14 ; 22)(\mathrm{q} 32.33 ; \mathrm{q} 13.31)]$. Array CGH analysis revealed a $6.6 \mathrm{Mb}$ deletion at 22q13.31qter. In case 7, a deletion of $5.0 \mathrm{Mb}$ was identified, whereas in case 8 the deletion spanned 3.9 Mb. The latter 22q13 deletion was associated with a duplication of $2.0 \mathrm{Mb}$, including 13 clones adjacent to the deleted region (Figure 2b). In addition to these subtelomeric copy number alterations, a duplication of $\sim 330 \mathrm{~kb}$ on $22 \mathrm{q} 11.1$ was detected in the same patient. Duplications in the same region next to the centromere were identified in two other cases in this series (cases 4 and 7). In the control panel of 72 normal individuals tested by a tiling resolution genome-wide BAC array, copy number 

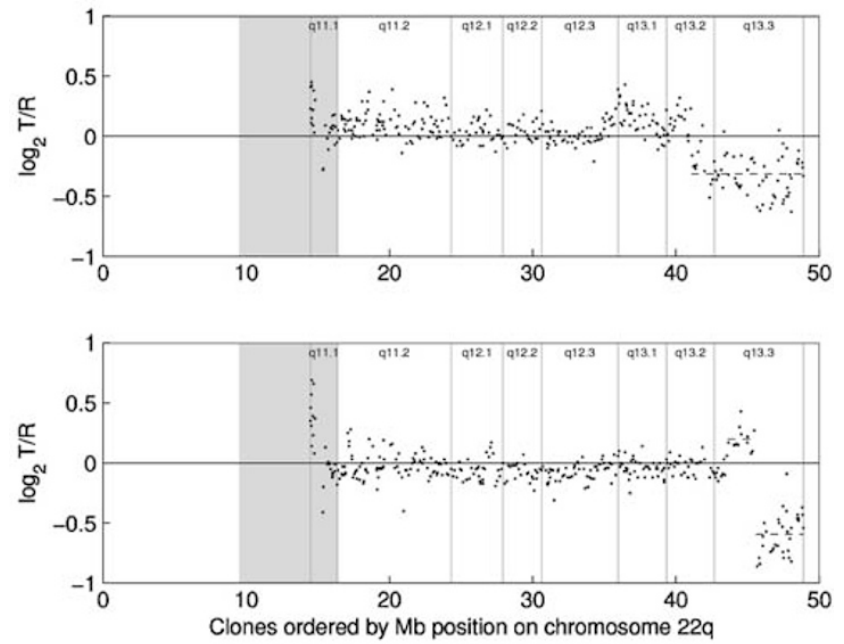

Figure 2 Examples of chromosome- 22 profiles obtained by array $\mathrm{CGH}$. Arrays contained 350 cloned chromosome- 22 genomic DNA targets (indicated by small circles representing the mean $\log _{2^{-}}$ transformed and Lowess-normalised $T / R$ intensity ratios), ordered from q11.1 to qter on the basis of physical mapping positions obtained from the May 2004 freeze of the UCSC Genome Browser. The centromere is indicated by a hatched area. A, case 1: del(22)(q13.2); $B$, case 8: del(22)(q13.31). Note the duplication of $2.0 \mathrm{Mb}$ on $22 q 13.31$ and the duplication on 22q11.1.

gain of the same region was observed in five individuals, whereas loss of the chromosomal segment was seen in 21 controls (de Vries, submitted).

\section{Discussion}

For detailed analyses of patients with known $22 \mathrm{q} 13$ deletions, a tiling resolution array was constructed with a 1.7-fold coverage of the long arm of chromosome 22 . Buckley et $a l^{12}$ demonstrated the utility of such a comprehensive chromosome 22 array by profiling acral melanoma, dermatofibrosarcoma, DiGeorge syndrome, and neurofibromatosis 2. The chromosome 22 tiling resolution array had an average clone spacing of $100 \mathrm{~kb}$, resulting in a resolution that is 30 times higher than highresolution karyotyping. The array did not cover the most telomeric $635 \mathrm{~kb}$ of the chromosome, which includes the gene SHANK3/ProSAP2. Haploinsufficiency of this gene has been proposed to be responsible for the major neurological features of the $22 \mathrm{q} 13$ deletion syndrome. ${ }^{6,13,14}$ However, FISH analysis using subtelomeric clones, confirmed the extension of all deletions found till the subtelomeric region.

In the present study, a considerable difference in deletion sizes was noted, which is in accordance with the results of Luciani et $\mathrm{al}^{3}$ which showed an extremely variable 22qter deletion size, extending from $160 \mathrm{~kb}$ to $9 \mathrm{Mb}$. Interestingly, a complex chromosome 22 rearrangement was observed in case 8 , with a unique combination of a deletion and a duplication of $22 \mathrm{q} 13$. The detection of the deletionduplication in this patient adds to a growing number of similar cases identified for other chromosomes, including deletion-duplications in $1 \mathrm{p}, 2 \mathrm{q}, 4 \mathrm{p}$, and $8 \mathrm{p} .^{15-18}$ Giglio et $a l^{19}$ demonstrated that the formation of the inverted duplications of $8 \mathrm{p}$ associated with a terminal deletion is caused by nonallelic homologous recombination (NAHR) between two olfactory receptor-gene clusters. In addition, Ballif et al described two terminal deletions of $1 \mathrm{p} 36$ associated with cryptic interrupted inverted duplications. This type of chromosome rearrangement may be more common than previously thought. The detection of cryptic duplication associated with terminal deletions is greatly improved by high-resolution copy number screening using array CGH. Further studies are needed to determine the mechanisms underlying these rearrangements. Ballif et al ${ }^{18}$ proposed a premeiotic model in which a terminally deleted chromosome is generated in the germ line and passes through at least one breakage-fusion-bridge cycle in which uncapped sister chromatids are fused by nonhomologous end joining (NHEJ), resulting in gametes with terminal deletions associated with cryptic interrupted inverted duplications. The identification of recurrent proximal breakpoints in cases 2 and 3 (between RP11786006 and RP11-236I15) and cases 5 and 6 (between RP11-564B15 and RP11-673D06), suggests that these regions may be prone to recombination, due to the presence of yet unknown genome architectural features. Segmental duplications were present at or close to the recurrent breakpoints and also to the other, nonrecurrent breakpoints in our series. To a large extent, chromosome rearrangement breakpoints are located in intervals containing complex genomic architecture, such as AT-rich palindromes or low-copy repearts (LCRs). ${ }^{20}$ Through the process of NAHR, LCRs can lead to translocations, inversions, duplications and interstitial deletions. ${ }^{21}$ However, the mechanisms for generating and/or stabilising terminally deleted chromosomes are poorly understood. NEHJ, possibly stimulated by LCRs or other repetitive sequences, may be one of the causative mechanisms for the terminal 22q13 deletions in our series.

In addition to the known 22qter deletions, a submicroscopic duplication on 22q11.1 next to the centromere was identified in three cases (cases 4, 7 and 8). In the control population of 72 normal individuals, copy number gains in the same region were identified in five individuals, whereas losses were observed in 21 controls (de Vries; submitted), indicating that this anomaly represents a LCV. This LCV at 22q11.1 was previously reported by Sebat et $a l^{22}$ (http://projects.tcag.ca/variation/) and stresses the variation of the human genome and the importance of parental and control analysis in case a submicroscopic alteration is identified. 


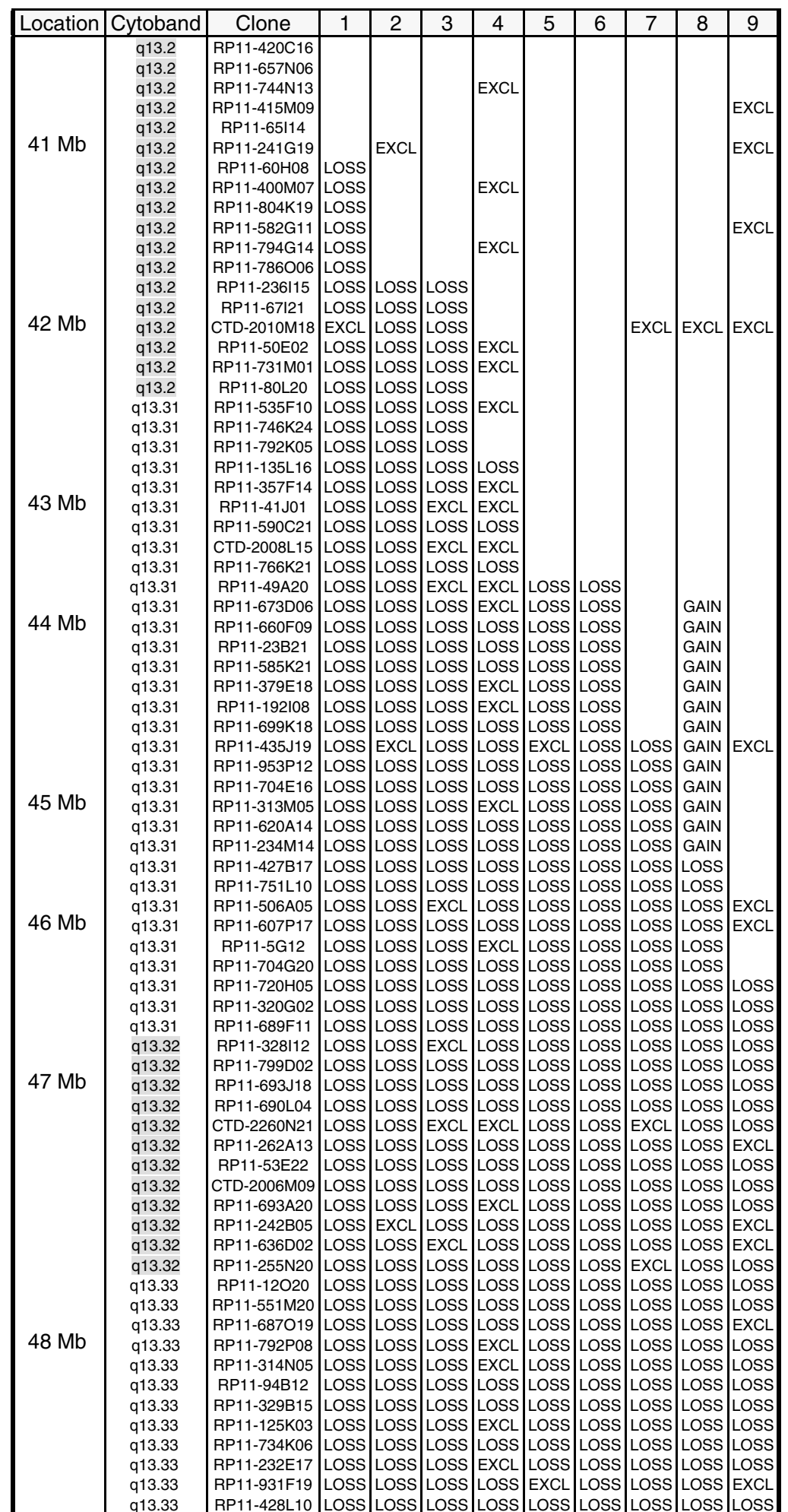

Figure 3 Overview of the array CGH results for the nine patients with monosomy 22qter. Arrays contained 350 chromosome- 22 DNA BAC clones, ordered from q11.1 to qter on the basis of physical mapping positions obtained from the May 2004 freeze of the UCSC Genome Browser. The distal $9 \mathrm{Mb}$ of the long arm of chromosome 22 is depicted in the figure. Copy number alterations were identified, using a Hidden Markov Model algorithm. Three hidden states represent normal (empty), loss (LOSS) and gain (GAIN) conditions. Individual clones that did not pass quality control criteria were excluded (EXCL). 
In the current study, no relation between clinical features and deletion size could be observed. The clinical features observed were consistent with the common clinical phenotype associated with the 22q13 deletion syndrome, ${ }^{5}$ although dolichocephaly and ptosis were not found in the current study. Global developmental delay and absent or severely delayed speech were observed in all patients. Hypotonia was present in $89 \%$ of the cases (8/9). A pointed chin was present in cases 1 and 4-7. Wilson et al previously suggested a candidate gene for this feature in the proximal region of $22 \mathrm{q} 13$, however this could not be confirmed in our series. In addition, in case 8 , ophthalmic assessment showed myopia and salt-and-pepper retinal changes suggestive of retinitis pigmentosa. These eye anomalies, which have not been reported in the $22 \mathrm{q} 13$ deletion syndrome before, are possibly attributable to the $2 \mathrm{Mb} 22 \mathrm{q} 13.31$ duplication in this patient. Relationship between the deletion size and clinical features could not be observed. However, case 9 with the smallest 22q13 deletion $(3.3 \mathrm{Mb})$, did not show any of the dysmorphic features commonly described in the 22q13 deletion syndrome. Facial dysmorphic features in this patient included upslanting palpebral fissures, a moderate hypertrophic nasal root, and thick lips. ${ }^{10}$ These findings underline the study of Wilson et al, in which no significant correlation with the size of the deletion could be demonstrated for most clinical features and support the idea that a gene in the $3.3 \mathrm{Mb}$ minimal deleted region (notably SHANK3/ ProSAP2) may be the major candidate gene in the $22 \mathrm{q} 13$ deletion syndrome. ${ }^{3-6}$

In conclusion, this study underscores the utility of array $\mathrm{CGH}$ for further characterisation of the size and nature of subtelomeric deletions. In addition, these results confirm the considerable differences in deletion size observed in patients with the $22 \mathrm{q} 13$ deletion syndrome, regardless of the clinical phenotype.

\section{Acknowledgements}

We thank the patients and their parents for their cooperation. David A Koolen, Joris A Veltman and Bert BA de Vries were supported by a grant from The Netherlands Organisation for Health Research and Development.

\section{References}

1 De Vries BB, Winter R, Schinzel A, Ravenswaaij-Arts C: Telomeres: a diagnosis at the end of the chromosomes. J Med Genet 2003; 40: $385-398$.

2 Knight SJ, Flint J: Perfect endings: a review of subtelomeric probes and their use in clinical diagnosis. J Med Genet 2000; 37: 401-409.

3 Luciani JJ, de Mas P, Depetris D et al: Telomeric 22q13 deletions resulting from rings, simple deletions, and translocations: cytogenetic, molecular, and clinical analyses of 32 new observations. J Med Genet 2003; 40: 690-696.

4 Manning MA, Cassidy SB, Clericuzio C et al: Terminal 22q deletion syndrome: a newly recognized cause of speech and language disability in the autism spectrum. Pediatrics 2004; 114: $451-457$.

5 Phelan MC, Rogers RC, Saul RA et al: 22q13 deletion syndrome. Am J Med Genet 2001; 101: 91-99.

6 Wilson HL, Wong AC, Shaw SR et al: Molecular characterisation of the 22q13 deletion syndrome supports the role of haploinsufficiency of SHANK3/PROSAP2 in the major neurological symptoms. J Med Genet 2003; 40: 575-584.

7 Veltman JA, Yntema HG, Lugtenberg D et al: High resolution profiling of $\mathrm{X}$ chromosomal aberrations by array comparative genomic hybridisation. J Med Genet 2004; 41: 425-432.

8 De Vries BB, Bitner-Glindzicz M, Knight SJ et al: A boy with a submicroscopic 22qter deletion, general overgrowth and features suggestive of FG syndrome. Clin Genet 2000; 58: 483-487.

9 Koolen DA, Nillesen WM, Versteeg MH et al: Screening for subtelomeric rearrangements in 210 patients with unexplained mental retardation using multiplex ligation dependent probe amplification (MLPA). J Med Genet 2004; 41: 892-899.

10 Goizet C, Excoffier E, Taine L et al: Case with autistic syndrome and chromosome 22q13.3 deletion detected by FISH. Am J Med Genet 2000; 96: 839-844.

11 Vissers LE, De Vries BB, Osoegawa K et al: Array-based comparative genomic hybridization for the genomewide detection of submicroscopic chromosomal abnormalities. Am J Hum Genet 2003; 73: 1261-1270.

12 Buckley PG, Mantripragada KK, Benetkiewicz $\mathrm{M}$ et al: A fullcoverage, high-resolution human chromosome 22 genomic microarray for clinical and research applications. Hum Mol Genet 2002; 11: 3221-3229.

13 Anderlid BM, Schoumans J, Anneren G et al: FISH-mapping of a $100-\mathrm{kb}$ terminal 22q13 deletion. Hum Genet 2002; 110: 439-443.

14 Bonaglia MC, Giorda R, Borgatti $\mathrm{R}$ et al: Disruption of the ProSAP2 gene in a $\mathrm{t}(12 ; 22)(\mathrm{q} 24.1 ; \mathrm{q} 13.3)$ is associated with the 22q13.3 deletion syndrome. Am J Hum Genet 2001; 69: 261-268.

15 Cotter PD, Kaffe S, Li L, Gershin IF, Hirschhorn K: Loss of subtelomeric sequence associated with a terminal inversion duplication of the short arm of chromosome 4. Am J Med Genet 2001; 102: 76-80.

16 Floridia G, Piantanida M, Minelli A et al: The same molecular mechanism at the maternal meiosis I produces mono- and dicentric 8p duplications. Am J Hum Genet 1996; 58: 785-796.

17 Bonaglia MC, Giorda R, Poggi G et al: Inverted duplications are recurrent rearrangements always associated with a distal deletion: description of a new case involving 2q. Eur J Hum Genet 2000; 8: 597-603.

18 Ballif BC, Yu W, Shaw CA, Kashork CD, Shaffer LG: Monosomy 1 p36 breakpoint junctions suggest pre-meiotic breakage-fusionbridge cycles are involved in generating terminal deletions. Hum Mol Genet 2003; 12: 2153-2165.

19 Giglio S, Broman KW, Matsumoto N et al: Olfactory receptor-gene clusters, genomic-inversion polymorphisms, and common chromosome rearrangements. Am J Hum Genet 2001; 68: 874-883.

20 Shaw CJ, Lupski JR: Implications of human genome architecture for rearrangement-based disorders: the genomic basis of disease. Hum Mol Genet 2004; 13 (Spec No 1): R57-R64.

21 Stankiewicz P, Lupski JR: Molecular-evolutionary mechanisms for genomic disorders. Curr Opin Genet Dev 2002; 12: 312-319.

22 Sebat J, Lakshmi B, Troge J et al: Large-scale copy number polymorphism in the human genome. Science 2004; 305: $525-528$. 